\title{
Airline Booking Limit Competition Game Under Differentiated Fare Structure
}

\author{
Joonkyum Lee, Sogang University, South Korea
}

Bumsoo Kim, Sogang University, South Korea

\begin{abstract}
We address a two-firm booking limit competition game in the airline industry. We assume aggregate common demand, and differentiated ticket fare and capacity, to make this study more realistic. A game theoretic approach is used to analyze the competition game. The optimal booking limits and the best response functions are derived. We show the existence of a pure Nash equilibrium and provide the closed-form equilibrium solution. The location of the Nash equilibrium depends on the relative magnitude of the ratios of the full and discount fares. We also show that the sum of the booking limits of the two firms remains the same regardless of the initial allocation proportion of the demand.
\end{abstract}

Keywords: Revenue Management; Booking Limit; Competition Game; Nash Equilibrium

\section{INTRODUCTION}

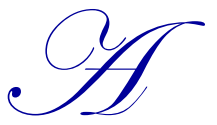

dvances in information technology have enabled people to access integrated airline booking information and have sensitized customers to more price-sensitive flight fares. Meanwhile, deregulation in the airline industry and the advent of discount airlines have brought about a more competitive environment in this industry, leading to its market expansion. Therefore, competition can considerably affect a firm's strategic and operational decision-making process, and airlines should take other players' actions into account.

Airline products tend to be less differentiated among airlines, leading to price competition. However, discount and full service airlines continue to co-exist in many markets, and there are differences in price and service among the same fare class. In addition, there has been a tendency for airlines to work within a common market given the prevalence of web-based travel agencies. While the ticket fare is the most critical factor for customers, some people prefer a particular airline owing to its frequent flyer program and service differentiation. Accordingly, a study on booking limit with competition under a common market and differentiated fare structure from the revenue management perspective could have substantial impact on both the academia and the industry.

Airline revenue management, sometimes called airline yield management, covers revenue factors (e.g., fare value structure), control system (e.g., booking limits and overbooking), and demand forecasting (e.g., cancellations) among others (McGill, \& Van Ryzin, 1999). We focus on the booking limit control system under competition in this study.

The booking limit decision is among the most significant issues in revenue management, and much research has been conducted on those issues. These aspects concern approving or denying a request for a certain fare class. The airline problem comprises multiple fare classes, causing demand and excess demand for each class, thus resulting in differentiated fare among players. A two-stage booking limit decision would be appropriate in such an environment. That is, players set the fares in the first stage, and then they observe the whole price information and set the booking limit in the second stage. This could be a good approximation of the current situation, where airlines publicize the fare information and then control their booking limit according to the market situation.

Even though the booking limit decision is the most important aspect in revenue management and competition is fierce, not much study has been devoted to booking limit policies considering competition. Netessine and Shumsky (2005) investigated horizontal and vertical competition for two airlines. They used firm-specific demand and the deterministic fraction of excess demand overflow to the other firm. They proved the existence of the Nash equilibrium, and the 
results indicated that more seats are protected for the full fare class. Song and Parlar (2012) studied a similar problem but they considered the penalty cost of rejected transfer reservations. They showed the uniqueness of the Nash equilibrium.

Li, Oum, and Anderson (2007) investigated two airlines with regard to competitive seat allocation for two fare classes. They used common market demand for players, and considered the initial allocation rule to be the proportional rationing rule. Demands for each fare class were not independent, but the fare structures were identical. They proved the existence of equilibrium with the same booking policy for full fare. Li, Zhang, and Zhang (2008) extended a previous study ( $\mathrm{Li}$ et al. 2007), which was based on cost asymmetry. Li et al. (2016) studied a duopoly game in revenue management with callable products. They showed that offering callable products can increase the booking limits but it might be detrimental to business.

Analogous problems of competition with inventories have been studied actively under the newsvendor setting. Parlar (1988) extended the classic newsvendor problem to the two-firm competitive newsvendor problem, in which the firms face independent random demands. Wang and Parlar (1994) examined three-player newsvendor competition and cooperation. Lippman and McCardle (1997) provided a generalized $n$-firm case analysis of the competitive newsvendor problem. They used the common market and examined four splitting rules. Netessine and Rudi (2003) carried out research on the competitive newsvendor problem for general $n$-firm cases. They used firm-specific demand, and the excess demand was reallocated with the deterministic fraction. Some of these studies proved the existence of the Nash equilibrium while others were implicit. The competitive newsvendor problem, however, differs from the booking limit problem because the airline problem has multiple fare classes and airplanes have limited capacity. Therefore, the results of the competitive newsvendor studies cannot be directly applied in the revenue management setting.

In analyzing booking limit competition, establishing the existence of a pure Nash equilibrium is important because it is operationally difficult to implement mixed strategies (Li, Oum, and Anderson, 2007). Lippman and McCardle (1997) use supermodularity to establish the existence of pure Nash equilibrium, and Netessine and Shumsky (2005) show the existence of a pure Nash equilibrium under mild assumptions. In this study, we show the existence of a pure Nash equilibrium by analyzing the response functions.

The above literature, however, did not consider the situation of the booking limit competition under aggregate common demand, distinct fare structure, and different capacity. Assuming a common market for airlines is more appropriate at this time given the prevalence of web-based travel agencies. The assumptions of distinct fare structures and different capacities (rather than identical ones) are also more realistic. Therefore, this study assumes aggregate demand, distinct fare structure, and different capacity. In doing so, it reflects a more realistic situation compared to existing studies that have considered competition.

In this study, we analyze the competitive booking limit decision under a common market and assuming distinct fare structures among airlines. Price and service differentiation and customer preference are reflected by the consumer choice model, such as the logit model, which determines the initial allocation and re-allocation of common demand. The optimal booking limit with competition is investigated and the existence of the Nash equilibrium based on the response functions is analyzed. To accomplish this, we use the game theoretic method to find equilibrium in competitive cases. This study provides a tractable and explicit solution, which can be extended to more complicated analyses. Most of the existing papers dealt with implicit solutions, but these cannot provide an intuitive answer for practical situations. We also discuss the characteristics of the equilibrium based on the closed-form solutions.

\section{MODEL}

\subsection{Setting and Notation}

We analyze the single-period single-leg airline market. The two airlines are indexed by $A$ and $B$ and provide flights for the same market. There are two fare classes, discount fare $(d)$ and full fare $(f)$, for each airline, denoted by $d^{A}$ and $d^{B}$, and $f^{A}$ and $f^{B}$ respectively. Capacities are denoted by $C^{A}$ and $C^{B}$. The demand for full fare is a random variable, $X$, and the demand for discount fare is large enough to fill the whole capacities. The probability distribution and 
cumulative distribution of demand are denoted by $g$ and $G$ respectively. The decision variables for airlines $A$ and $B$ are the protection levels for the full fare class, denoted by $y^{A}$, and $y^{B}$ respectively. The objective of each airline is to set a number of seats for the full fare class so as to maximize the expected revenue, taking the other player's decision into account.

McGill and Van Ryzin's (1999) six common assumptions of seat inventory control are also applied to this model: 1) sequential booking classes, 2) low-before-high fare booking arrival pattern, 3) statistical independence of demand between booking classes, 4) no cancellation or no-shows (no overbooking), 5) single-flight leg with no network effect, 6) no batch booking. These assumptions are considerably common in the revenue management literature, and we also adopt them for the purpose of tractability of complex problems. The model in this study assumes a two-stage game, which means the fares will be determined at the first stage, and the airline will decide the booking limit after observing the other players' fare decision.

\subsection{Demand Allocation}

As described above, we assume a common market for all players, which is more realistic and simple. Total demand is a random variable denoted by $X$ and follows an arbitrary distribution. For initial allocation, the fixed demand fraction coefficient for airline $A$ and $B$ is $\alpha$ and $(1-\alpha)$ respectively. Therefore, the initial demand for airline $A$ is $\alpha \mathrm{X}$, and that for airline $B$ is $(1-\alpha) \mathrm{X}$. In this case, the demand for both airlines is perfectly correlated.

Excess demand, namely demand that cannot be satisfied by initial allocation, is reallocated to the other airline. This means that the customer who cannot buy a ticket of his or her favorite airline will search for another airline as a substitute. We assume that all the excess demand overflows to the other airline.

We also assume the existence of price and other differentiation features, including frequent flight programs and service quality. These factors as well as the ticket fare will affect the demand allocation proportion, $\alpha$. The value of $\alpha$ can be determined according to commonly used choice models such as the logit model. If the logit model is used to capture the customer choice decision, $\alpha$ is determined as follows.

$$
\alpha=\frac{K^{A} \exp \left(-f^{A} / \mu\right)}{1+K^{A} \exp \left(-f^{A} / \mu\right)+K^{B} \exp \left(-f^{B} / \mu\right)}, \quad \mu \geq 0
$$

The attractiveness of product features, including frequent flight programs and service quality, are captured by $K^{A}$ and $K^{B} . \mu$ is a scaling factor. The value 1 in the denominator denotes external alternatives (e.g., train or bus), which means that the size of the whole market could be affected by the fares, and if the airline fare is too high, then people will choose other options. Therefore, the decision on the ticket fares can change the size of the market. In this study, the fares are presumed to be predetermined.

\section{ANALYSIS AND RESULTS}

\subsection{Revenue Function}

The total revenue for airline $A$ comprises revenues from the full fare class and the discount fare class.

$$
\Pi^{A}=f^{A} \min \left[\alpha X+\left[(1-\alpha) X-y^{B}\right]^{+}, y^{A}\right]+d^{A}\left(C^{A}-y^{A}\right)
$$

Let $R^{A}=\alpha X+\left[(1-\alpha) X-y^{B}\right]^{+}$, and $T^{A}=\min \left[\alpha X+\left[(1-\alpha) X-y^{B}\right]^{+}, y^{A}\right]$. The effective demand for airline $A$, represented by $R^{A}$, is the sum of the initial demand for airline $A$ itself and the overflow from the excess demand from airline $B$. If airline $B$ 's protection level for the full fare is less than the initial demand for airline $B$, there will be positive overflow demand from $B$ to $A$, and all the excess customers will try to buy tickets from airline $A$. Note that the effective demand for $A$ depends on the other player's decision variable. $T^{A}$ is the actual number of full fare customers of airline $A$, which is the minimum of the effective demand and protection levels. The rest of the capacity will be filled by discount fare customers. 
The value of $R^{A}$ varies according to the critical value of $y^{B} /(1-\alpha)$ : if $x>y^{B} /(1-\alpha)$, then $R^{A}=x-y^{B}$; if $x \leq$ $y^{B} /(1-\alpha)$, then $R^{A}=\alpha x$. Similarly, the value of $T^{A}$ is dependent on the critical values of $y^{A}+y^{B}$, and $y^{A} / \alpha$.

It can be shown that the realized values of $R^{A}$ and $T^{A}$ vary depending on the critical values of $y^{B} /(1-\alpha), y^{A}+y^{B}$, and $y^{A} / \alpha$. The critical values identify the critical point where demand $X$ reaches protection levels. To be specific, the demand reaches airline $A$ 's protection level at $y^{A} / \alpha$, airline $B$ 's protection level at $y^{b} /(1-\alpha)$, and sum of the both protection levels at $y^{A}+y^{B}$. After the demand exceeds $y^{A}+y^{B}$, both airlines' full fare classes will be fully filled. Depending on the value of $\alpha$, and the critical values, $R^{A}$ and $T^{A}$ are determined as follows.

Case 1: If $\alpha \geq y^{A} /\left(y^{A}+y^{B}\right)$, then $y^{A} / \alpha \leq y^{A}+y^{B} \leq y^{B} /(1-\alpha)$

\begin{tabular}{|c|c|c|c|}
\hline & \multicolumn{1}{|c|}{$y^{A} / \alpha$} & $y^{A}+y^{B} /(1-\alpha)$ \\
\hline$R^{A}$ & $\alpha x$ & $y^{A}$ & $x-y^{B}$ \\
\hline$T^{A}$ & $\alpha x$ & $y^{A}$ & \\
\hline
\end{tabular}

Case 2: If $\alpha<y^{A} /\left(y^{A}+y^{B}\right)$, then $y^{B} /(1-\alpha) \leq y^{A}+y^{B} \leq y^{A} / \alpha$

\begin{tabular}{|l|l|l|l|}
\hline & $y^{B} /(1-\alpha)$ & $y^{A}+y^{B}$ \\
\hline$R^{A}$ & $x \longleftarrow$ & $x-y^{B}$ \\
\hline$T^{A}$ & $\alpha x$ & & $y^{A}$ \\
\hline
\end{tabular}

\subsection{Maximizing Expected Revenue}

To find the optimal protection level that maximizes the expected revenue, we find the first-order condition using Leibniz's rule.

$$
E\left[\Pi^{A}\right]=f^{A} \min \left[\alpha x+\left[(1-\alpha) x-y^{B}\right]^{+}, y^{A}\right]+d^{A}\left(C^{A}-y^{A}\right)=f^{A} E\left[T^{A}\right]+d^{A}\left(C^{A}-y^{A}\right)
$$

Therefore, $\partial E\left[\Pi^{A}\right] / \partial y^{A}=f^{A} \partial E\left[T^{A}\right] / \partial y^{A}-d^{A}$.

For Case $1, E\left[T^{A}\right]=\int_{0}^{y^{A} / \alpha} \alpha x g(x) d x+\int_{y^{A} / \alpha}^{\infty} y^{A} g(x) d x$.

$$
\frac{\partial E\left[T^{A}\right]}{\partial y^{A}}=\alpha \frac{y^{A}}{\alpha} \frac{1}{\alpha} g\left(\frac{y^{A}}{\alpha}\right)-y^{A} g\left(\frac{y^{A}}{\alpha}\right) \frac{1}{\alpha}+\int_{y^{A} / \alpha}^{\infty} g(x) d x=\int_{y^{A} / \alpha}^{\infty} g(x) d x=1-G\left(y^{A} / \alpha\right)
$$

Thus, $\partial E\left[\Pi^{A}\right] / \partial y^{A}=f^{A}\left(1-G\left(y^{A} / \alpha\right)\right)-d^{A}$. The first-order condition is $G\left(y^{A} / \alpha\right)=1-d^{A} / f^{A}$. Hence, the optimal protection level of airline $A$ is $y^{A}=\alpha G^{-1}\left(1-d^{A} / f^{A}\right)$. Note that $1-d^{A} / f^{A}>0$ by assumption. We assume that the demand function is monotonically increasing so that the second-order condition is satisfied.

For Case 2, $E\left[T^{A}\right]=\int_{0}^{y^{B} /(1-\alpha)} \alpha x g(x) d x+\int_{y^{B} /(1-\alpha)}^{y^{A}+y^{B}}\left(x-y^{B}\right) g(x) d x+\int_{y^{A}+y^{B}}^{\infty} y^{A} g(x) d x$.

$$
\frac{\partial E\left[T^{A}\right]}{\partial y^{A}}=\int_{y^{A}+y^{B}}^{\infty} g(x) d x=1-G\left(y^{A}+y^{B}\right)
$$

Thus, $\partial E\left[\Pi^{A}\right] / \partial y^{A}=f^{A}\left(1-G\left(y^{A}+y^{B}\right)\right)-d^{A}$. The first-order condition is $G\left(y^{A}+y^{B}\right)=1-d^{A} / f^{A}$. Hence, the optimal protection level of airline $A$ is $y^{A}=G^{-1}\left(1-d^{A} / f^{A}\right)-y^{B}$. 
We can find the optional protection level of airline $B$ in a similar way. The optimal protection levels are summarized in Table 1.

Table 1. The optimal protection level for each airline

\begin{tabular}{l|l|l}
\hline & \multicolumn{1}{c}{ If $\boldsymbol{\alpha} \geq \boldsymbol{y}^{\boldsymbol{A}} /\left(\boldsymbol{y}^{\boldsymbol{A}}+\boldsymbol{y}^{\boldsymbol{B}}\right)$} & \multicolumn{1}{c}{ If $\boldsymbol{\alpha}<\boldsymbol{y}^{\boldsymbol{A}} /\left(\boldsymbol{y}^{\boldsymbol{A}}+\boldsymbol{y}^{\boldsymbol{B}}\right)$} \\
\hline Optimal protection level of $A$ & $y^{A}=\alpha G^{-1}\left(1-d^{A} / f^{A}\right)$ & $y^{A}=G^{-1}\left(1-d^{A} / f^{A}\right)-y^{B}$ \\
\hline Optimal protection level of $B$ & $y^{B}=G^{-1}\left(1-d^{B} / f^{B}\right)-y^{A}$ & $y^{B}=(1-\alpha) G^{-1}\left(1-d^{B} / f^{B}\right)$ \\
\hline
\end{tabular}

\subsection{Best Response Function and Nash Equilibrium}

The above optimal protection levels are the best response of each firm to the other firm's action. Furthermore, if their beliefs of other firms' action correspond to their actual action, the pair of their protection levels should be a pure Nash equilibrium. Figure 1 illustrates the best responding functions.

The Nash equilibrium is the intersection of these two best response functions. Table 2 shows a Nash equilibrium denoted by $\bar{y}^{A}$ and $\bar{y}^{B}$ for airlines $A$ and $B$ respectively.

Table 2. The Nash equilibrium solution

\begin{tabular}{l|l|l}
\hline & \multicolumn{1}{c}{ If $\frac{\boldsymbol{d}^{A}}{\boldsymbol{f}^{\boldsymbol{A}}}<\frac{\boldsymbol{d}^{B}}{\boldsymbol{f}^{\boldsymbol{B}}}$} & \multicolumn{1}{c}{ If $\frac{\boldsymbol{d}^{A}}{\boldsymbol{f}^{\boldsymbol{A}}} \geq \frac{\boldsymbol{d}^{\boldsymbol{B}}}{\boldsymbol{f}^{\boldsymbol{B}}}$} \\
\hline Nash equilibrium $\bar{y}^{A}$ & $\alpha G^{-1}\left(1-\frac{d^{A}}{f^{A}}\right)$ & $G^{-1}\left(1-\frac{d^{A}}{f^{A}}\right)-(1-\alpha) G^{-1}\left(1-\frac{d^{B}}{f^{B}}\right)$ \\
\hline Nash equilibrium $\bar{y}^{B}$ & $G^{-1}\left(1-\frac{d^{B}}{f^{B}}\right)-\alpha G^{-1}\left(1-\frac{d^{A}}{f^{A}}\right)$ & $(1-\alpha) G^{-1}\left(1-\frac{d^{B}}{f^{B}}\right)$ \\
\hline
\end{tabular}

The best response function of each airline is piece-wise linear. For example, the best response of $B$ is to decrease the booking limit up to the threshold and to maintain it after the threshold as $A$ increases the booking limit. As $A$ increases the booking limit, the probability that $B$ has some overflow demand decreases. Therefore, $B$ has to decrease the booking limit. However, after the threshold, the expected revenue from the overflow full fare customers exceeds the expected revenue from the discount fare, and therefore, it has to maintain the booking limit.

There exists a pure Nash equilibrium booking limit solution. The location of the solution depends on the relative magnitude of the ratios of the discount and the full fare. If the ratios are the same, then $\bar{y}^{A}=\alpha G^{-1}(1-d / f)$ and $\bar{y}^{B}=(1-\alpha) G^{-1}(1-d / f)$, which means that both airlines' booking limits are proportional to their own demand. However, if the ratio of airline $A$ is smaller, or the full fare of $A$ is relatively expensive, then the optimal booking limit of airline $B$ will increase at the equilibrium.

Note that $\bar{y}^{A}+\bar{y}^{B}=\max \left\{G^{-1}\left(1-d^{A} / f^{A}\right), G^{-1}\left(1-d^{B} / f^{B}\right)\right\}$, and it does not depend on the value of $\alpha$. That is, the total booking limit is independent of the proportions of the demand for $A$ and $B$. Therefore, regardless of the initial demand allocation, airline $A$ will reduce the booking limit if $B$ increases the booking limit, and the magnitude of the increase and the decrease will be the same. 
Figure 1. The best response functions of each airline and the Nash equilibrium

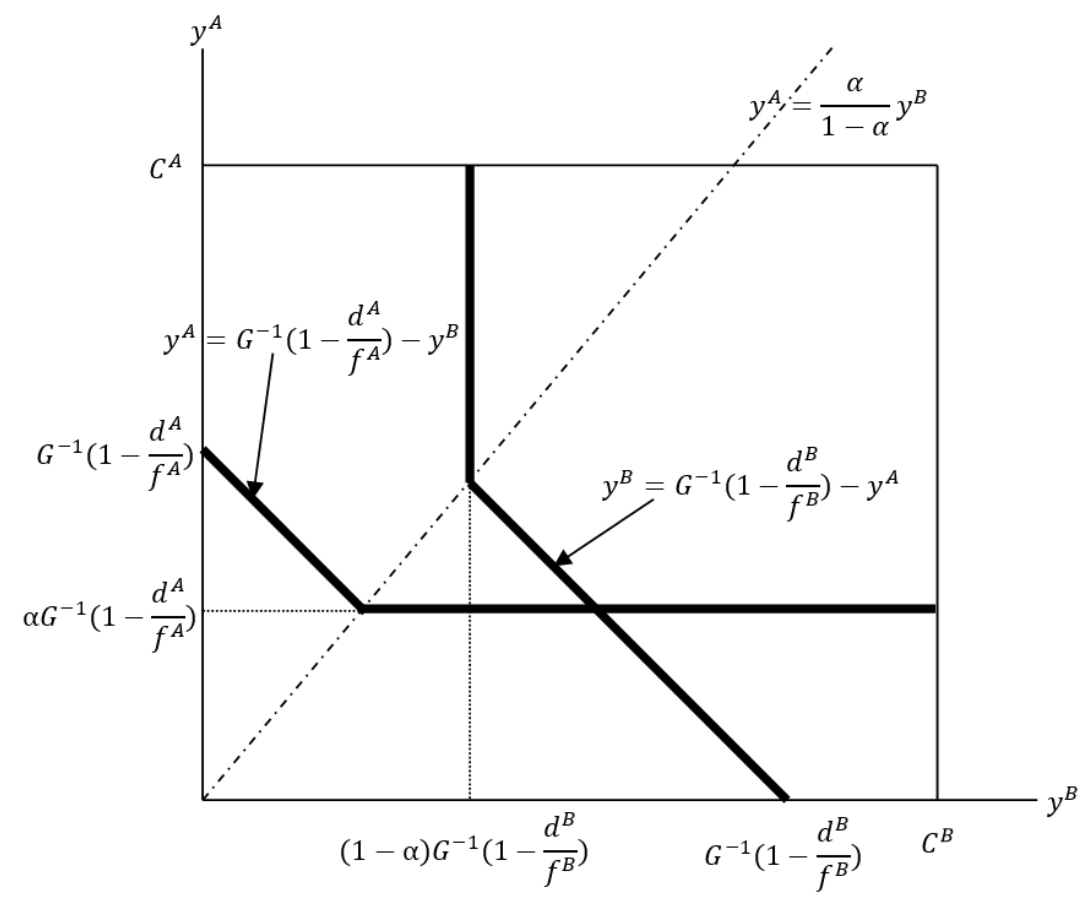

\section{CONCLUSION}

Nowadays, the airline industry faces a competitive environment. The prevalence of web-based agencies has resulted in a common market for customers. Companies in the same sector compete with each other with regard to ticket fares and booking limits. The excess demand from a firm will overflow to the other firm. Nevertheless, there is a lack of studies on the competitive booking limit game. Unlike the existing literature, we consider a more realistic situation: aggregate common demand, and distinct fare structure as well capacity for each airline. We use a game theoretic approach to analyze a two-firm booking limit competition game. We find the optimal booking limit and the best response function for each firm. We show that there exists a pure Nash equilibrium, which depends on the relative magnitude of the ratios of the full and discount fares. We also show that the sum of the booking limits of the two firms remains the same regardless of the initial allocation proportion of the demand.

This paper provides tractable and closed-form solutions of the pure Nash equilibrium, which can be extended to more complicated analyses. We only consider two-firm competition but competition among an arbitrary number of firms could give more general insights. We fix the full and discount fares in this study but analyzing simultaneous decisions about fares and booking limits would be of interest to researchers and practitioners. Finally, we do not consider dynamic pricing and the booking limit decision. Studies on those topics will provide more realistic lessons.

\section{AUTHOR BIOGRAPHIES}

Joonkyum Lee is an Assistant Professor at Sogang University. His research focus is on operations management and supply chain management. He received his Ph.D. from the Samuel Curtis Johnson Graduate School of Management at Cornell University. E-mail: jklee@sogang.ac.kr

Bumsoo Kim is an Assistant Professor at Sogang University. His research focus is on Bayesian statistical modelling and decision analysis. He earned his Ph.D. from George Washington University School of Business. E-mail: bk4498@sogang.ac.kr (corresponding author) 


\section{REFERENCES}

Li, M. Z., Oum, T. H., \& Anderson, C. K. (2007). An airline seat allocation game. Journal of Revenue and Pricing Management, $6(4), 321-330$.

Li, M. Z., Zhang, A., \& Zhang, Y. (2008). Airline seat allocation competition. International Transactions in Operational Research, 15(4), 439-459.

Li, T., Xie, J., Lu, S., \& Tang, J. (2016). Duopoly game of callable products in airline revenue management. European Journal of Operational Research, 254(3), 925-934.

Lippman, S. A., \& McCardle, K. F. (1997). The competitive newsboy. Operations research, 45(1), 54-65.

McGill, J. I., \& Van Ryzin, G. J. (1999). Revenue management: Research overview and prospects. Transportation science, 33(2), 233-256.

Netessine, S., \& Rudi, N. (2003). Centralized and competitive inventory models with demand substitution. Operations Research, 51(2), 329-335.

Netessine, S., \& Shumsky, R. A. (2005). Revenue management games: Horizontal and vertical competition. Management Science, 51(5), 813-831.

Parlar, M. (1988). Game theoretic analysis of the substitutable product inventory problem with random demands. Naval Research Logistics, 35(3), 397-409.

Song, J., \& Parlar, M. (2012). Analysis of a simple capacity game. International Transactions in Operational Research, 19(3), 435-461.

Wang, Q., \& Parlar, M. (1994). A three-person game theory model arising in stochastic inventory control theory. European Journal of Operational Research, 76(1), 83-97. 
NOTES 